\title{
In vitro Expansion of Umbilical Cord Blood Derived Mesenchymal Stem Cells (UCB-MSCs) Under Hypoxic Conditions
}

\author{
Jungyun Yang ${ }^{1,2}$, Jihye Kwon ${ }^{2}$, Miyeon Kim², Yunkyung Bae', Hyejin $\mathrm{Jin}^{2}$, \\ Hohyun Park ${ }^{3}$, Young Woo Eom ${ }^{4}$ and Ki-Jong Rhee ${ }^{1, \dagger}$ \\ ${ }^{l}$ Department of Biomedical Laboratory Science, College of Health Sciences, Yonsei University at Wonju, \\ Wonju, Gangwon-do 220-710, Korea \\ ${ }^{2}$ Biomedical Research Institute, MEDIPOST Co.Ltd., Seongnam, Gyeonggi-do 463-400, Korea \\ ${ }^{3}$ Department of Biomedical Laboratory Science, Mokpo Science University, Mokpo-si, Jeollanam-do 530-730, Korea \\ ${ }^{4}$ Cell Therapy and Tissue Engineering Center, Wonju College of Medicine, Yonsei University at Wonju, \\ Wonju, Gangwon-do 220-701, Korea
}

\begin{abstract}
Mesenchymal stem cells (MSCs) have the ability to self-renew and differentiate into multi-lineage cells, thus highlighting the feasibility of using umbilical cord blood-derived MSCs (UCB-MSCs) for cell-therapy and tissueengineering. However, the low numbers of UCB-MSC derived from clinical samples requires that an ex vivo expansion step be implemented. As most stem cells reside in low oxygen tension environments (i.e., hypoxia), we cultured the UCBMSCs under $3 \% \mathrm{O}_{2}$ or $21 \% \mathrm{O}_{2}$ and the following parameters were examined: proliferation, senescence, differentiation and stem cell specific gene expression. UCB-MSCs cultured under hypoxic conditions expanded to significantly higher levels and showed less senescence compared to UCB-MSCs cultured under normoxic conditions. In regards to differentiation potential, UCB-MSCs cultured under hypoxic and normoxic conditions both underwent similar levels of osteogenesis as determined by ALP and von Kossa assay. Furthermore, UCB-MSCs cultured under hypoxic conditions exhibited higher expression of OCT4, NANOG and SOX2 genes. Moreover, cells expanded under hypoxia maintained a stem cell immnunophenotype as determined by flow cytometry. These results demonstrate that the expansion of human UCB-MSCs under a low oxygen tension microenvironment significantly improved cell proliferation and differentiation. These results demonstrate that hypoxic culture can be rapidly and easily implemented into the clinical-scale expansion process in order to maximize UCB-MSCs yield for application in clinical settings and at the same time reduce culture time while maintaining cell product quality.
\end{abstract}

Key Words: Umbilical cord blood, Mesenchymal stem cell, Hypoxia

\section{INTRODUCTION}

Mesenchymal stem cells (MSCs) exhibit capacity for

\footnotetext{
* Received: February 28, 2015 / Accepted: March 24, 2015

${ }^{\dagger}$ Corresponding author: Ki-Jong Rhee. Department of Biomedical Laboratory Science College of Health Sciences, Yonsei University at Wonju Wonju, Gangwon-do 220-710, Korea.

Tel: +82-33-760-2445, Fax: +82-33-760-2561

e-mail: kjrhee@yonsei.ac.kr

(C)The Korean Society for Biomedical Laboratory Sciences. All rights reserved.
}

self-renewal and multi-lineage differentiation and thus are a useful resource for tissue engineering and regenerative medicine (Caplan, 2007). MSCs are generally obtained surgically from adult bone marrow and adipose tissues. However, the age of the donor is a critical factor with tissues obtained from older patients having less robust growth characteristics (Fukuchi et al., 2004; Kern et al., 2006; Mosna et al., 2010; Alves et al., 2012). To circumvent these issues, tissues from other sources such as the placenta, umbilical cord blood, and amnion have been considered as 
alternative sources (Kern et al., 2006; Lu et al., 2006; Reinisch et al., 2007; Han et al., 2008). Of these tissues, the umbilical cord collected after birth is a discarded tissue and therefore lacks ethical considerations and the collection of UCB is comparably simple compared to other tissues. In addition, compared to bone marrow derived MSCs, UCBMSCs are less differentiated, does not easily differentiate into cancer cells, exhibits tolerance in recipients and undergoes less senescence (Kern et al., 2006; Flynn et al., 2007; Reinisch et al., 2007; Oh et al., 2008; Jin et al., 2013). For these reasons, UCB-MSCs are more clinically useful in the clinical setting. However, UCB-MSCs have not been investigated as extensively compared to bone marrow and adipose tissues and the isolation is considered to be less efficient compared to bone marrow and adipose tissues (Erices et al., 2000).

For successful therapy, a high number of stem cells are required and thus the initially isolated MSCs must undergo cell expansion in vitro prior to injection into patients. However, during the in vitro expansion process, MSCs gradually lose their proliferative capacity, self-renewing ability, differentiation potential and have a tendency to undergo cellular senescence (Jo et al., 2008; Kretlow et al., 2008). Thus, a balance between cell expansion and maintenance of "stemness" is required. Expansion of stem cells in vitro requires optimal concentrations of nutrients, growth factors, oxygen and likely other less defined ingredients. Of these parameters, oxygen has been considered one key consideration during in vitro expansion. Traditionally, cell cultures are performed under normoxic conditions (21\% $\mathrm{O}_{2}$ ). However, it was found that the stem cell niche in vivo is relatively a hypoxic environment $\left(1 \sim 9 \% \mathrm{O}_{2}\right)$ thus demonstrating that the in vitro cell culture conditions are not faithfully mimicking the in vivo situation (Cipolleschi et al., 1993; Braun et al., 2001). Recently, improved culture techniques have elevated the recovery rate of UCB-MSCs to $65 \%$ (Lee et al., 2004; Avanzini et al., 2009; Fan et al., 2009). Furthermore, many studies have found that culturing MSCs from bone marrow and adipose tissue under hypoxic conditions improved yield in terms of proliferation, senescence, differentiation and self-renewal (Wang et al., 2005; Grayson et al., 2006; Grayson et al., 2007). However, little is known about the growth characteristics of UCB-MSCs when cultured in hypoxic conditions.

In the current study, we cultured UCB-MSCs under hypoxic conditions and compared the stem cell potential of these cells compared UCB-MSCs cultured under normoxic conditions. We assessed the cell growth proliferation rate, senescence, cell phenotype, differentiation potential and expression of stem cell specific genes. We found that UCBMSCs cells cultured under normoxic conditions exhibited better growth capacity, higher differentiation potential while maintaining "stemness" compared to cells expanded under normoxic conditions.

\section{MATERIAL AND METHODS}

\section{Isolation and Culture of MSCs}

Human full-term UCB samples were collected from umbilical cord veins from five women immediately after vaginal birth (mean age: 29.4 years, range 22 32 years). The sexes of the neonates were 2 males and 3 females. This study was approved by the Institutional Review Board at Medipost and informed consent was obtained from all subjects. The umbilical cord was ligated and placed in citrate phosphate dextrose adinime (CPDA-1) and thereafter processed within 40 hours. The UCB was separated using Ficoll-Hypaque (Sigma, St. Louis, USA) by centrifugation and mononuclear cells cultured in $\alpha$-MEM (Gibco, USA) containing $10 \% \mathrm{FBS}$ at a concentration of $5 \times 10^{5} / \mathrm{cm}^{2}$. The cells were initially cultured at $37^{\circ} \mathrm{C}$ in a humidified atmosphere of $5 \% \mathrm{CO}_{2}$. Cell culture media was exchanged twice a week. When MSCs reached $\sim 80 \%$ confluency, cells were trypsinized and reseeded at $2 \times 10^{3} / \mathrm{cm}^{2}$. Cells from the sixth subculture, hereafter referred to as P6 (passage 6), were used for subjected to proliferation or differentiation experiments.

\section{Assessment of cell proliferation}

Cell proliferation was assessed using trypan blue dye exclusion assay. Cells were trypsinized, mixed with trypan blue dye and viable cells enumerated using a hemacytometer. The formula for CPD is as follows: $\mathrm{CPD}=\log \left(N / N_{0}\right) / \log 2$ ( $N$ : Total cell number recovered; $N_{0}$ : Total cell number at P6). 


\section{Surface phenotypic analysis}

Cells were trypsinized, fixed with $1 \%$ paraformaldehyde in PBS and stained with fluorochrome-labeled antibodies for $15 \mathrm{~min}$, washed and analyzed with a flow cytometer (FACsCalibur, BD Bioscience, USA). The antibodies used are as follows: anti-CD14-FITC (Becton-Dickinson, San Jose, CA, USA), anti-CD45-FITC (Becton-Dickinson), anti-HLADR-FITC (Becton-Dickinson), anti-CD29-PE (Pharmingen, Los Angeles, CA, USA), anti-CD73-PE (Pharmingen), antiCD90-PE (Pharmingen), anti-CD166 (Pharmingen) and anti-CD105-PE (Serotec, Kidlington, UK). Cells were also stained with the appropriate fluorochrome-labeled isotype matched antibodies.

\section{Osteogenic differentiation}

To induce osteogenic differentiation, MSCs were plated $\left(100 \sim 300\right.$ cells $\left./ \mathrm{cm}^{2}\right)$ in osteogenic differentiation medium $(\alpha$-MEM, 10\% FBS, $0.1 \mu \mathrm{M}$ dexamethasone, $10 \mathrm{mM} \beta$ glycerol phosphate, $50 \mu \mathrm{M}$ L-ascorbic acid 2-phosphate) for 2 weeks. Media was changed twice weekly. Differentiation into osteocytes was confirmed using von Kossa stain and alkaline phosphatase (ALP) staining. For von Kossa staining, methanol-fixed cells were treated with silver nitrate and exposed to ultraviolet light for $1 \mathrm{hr}$. Then, cells were stained with $5 \%$ sodium thiosulfate for $2 \mathrm{~min}$ and then counterstained with Mayer's hematoxylin. For ALP staining, cells were fixed with $60 \%$ acetone, stained with naphthanol AS-MX phosphate solution containing fast violet B salt for $30 \mathrm{~min}$ at room temperature and then counterstained with Mayer's hematoxylin.

\section{Assessment of cellular senescence}

To detect cells undergoing senescence, cells which showed $<2$-fold doubling during 1 week of culture were plated at a density of $7 \times 10^{4} /$ well in a 6-well plate for 1 day and then stained with Senescence $\beta$-galactosidase Staining Kit ${ }^{\circledR}$ (Cell Signaling Technology). Stained cells were enumerated under a microscope and represented as \% of total cells.

\section{Real-time RT-PCR}

Total RNA were extracted with TRIzol ${ }^{\circledR}$ (Invitrogen,
Grand Island, New York, USA). cDNA was synthesized using $1 \mu \mathrm{g}$ of RNA, Superscript II Reverse Transcriptase (Invitrogen) according to the manufacturer's methods. Realtime PCR analysis was performed using LightCycler TM 480 (Roche, Mannheim, Germany) using SYBR green and specific PCR primers. The primers were designed using the Universal Probe Library Assay Design Center (Roche) database. The primer sequences are as follows: $\beta$-actin (forward) 5'-CCAACCGCGAGAAGATGA-3', (reverse) 5'-CCAGAGGCGTACAGGGATAG-3', OCT-4 (forward) 5'-CAATTTGCCAAGCTCCTGA-3', (reverse) 5'-CGTTTGGCTGAATACCTTCC-3', NANOG (forward) 5'-AGATGCCTCACACGGAGACT-3', (reverse) 5'-TTTGCGACACTCTTCTCTGC-3', SOX-2 (forward) 5'-TGCTGCCTCTTTAAGACTAGGAC-3', (reverse) 5'-CCTGGGGCTCAAACTTCTCT-3'. Relative mRNA levels were compared using the comparative threshold cycle $\left(2^{-\Delta \Delta C t}\right)$ method. The $\beta$-actin mRNA levels from normoxic cells were set as 1 .

\section{Statistical analysis}

Paired Student's $t$-test and one-way ANOVA was used for statistical analysis. Data are shown as the mean \pm SD from three independent experiments. Graphs were generated using Sigma Plot. $P<0.05$ denote statistical significance.

\section{RESULTS}

\section{Ex vivo expansion of UCB-MSC cultured under hypoxia and normoxia}

Rapid and prolonged cell expansion is a critical issue when preparing UCB-MSCs for regenerative medicine. We assessed the cell proliferative capacity of five UCB-MSC samples at $\mathrm{P} 6$ cultured under hypoxic $\left(3 \% \mathrm{O}_{2}\right)$ and normoxic $\left(21 \% \mathrm{O}_{2}\right)$ conditions. We found that with the exception of one sample (UCB-MSC-5), four samples showed increased CPD during hypoxic culture compared to normoxic culture (Fig. 1A). UCB-MSC-5 grew exceptionally well under both conditions and thus no difference was noted. The cumulative cell number at P10 of hypoxic culture indicates that UCBMSCs expanded approximately 50 100\% more compared to UCB-MSCs cultured under normoxic conditions (Fig. 1B). Although the extent of proliferation was varied among 
A

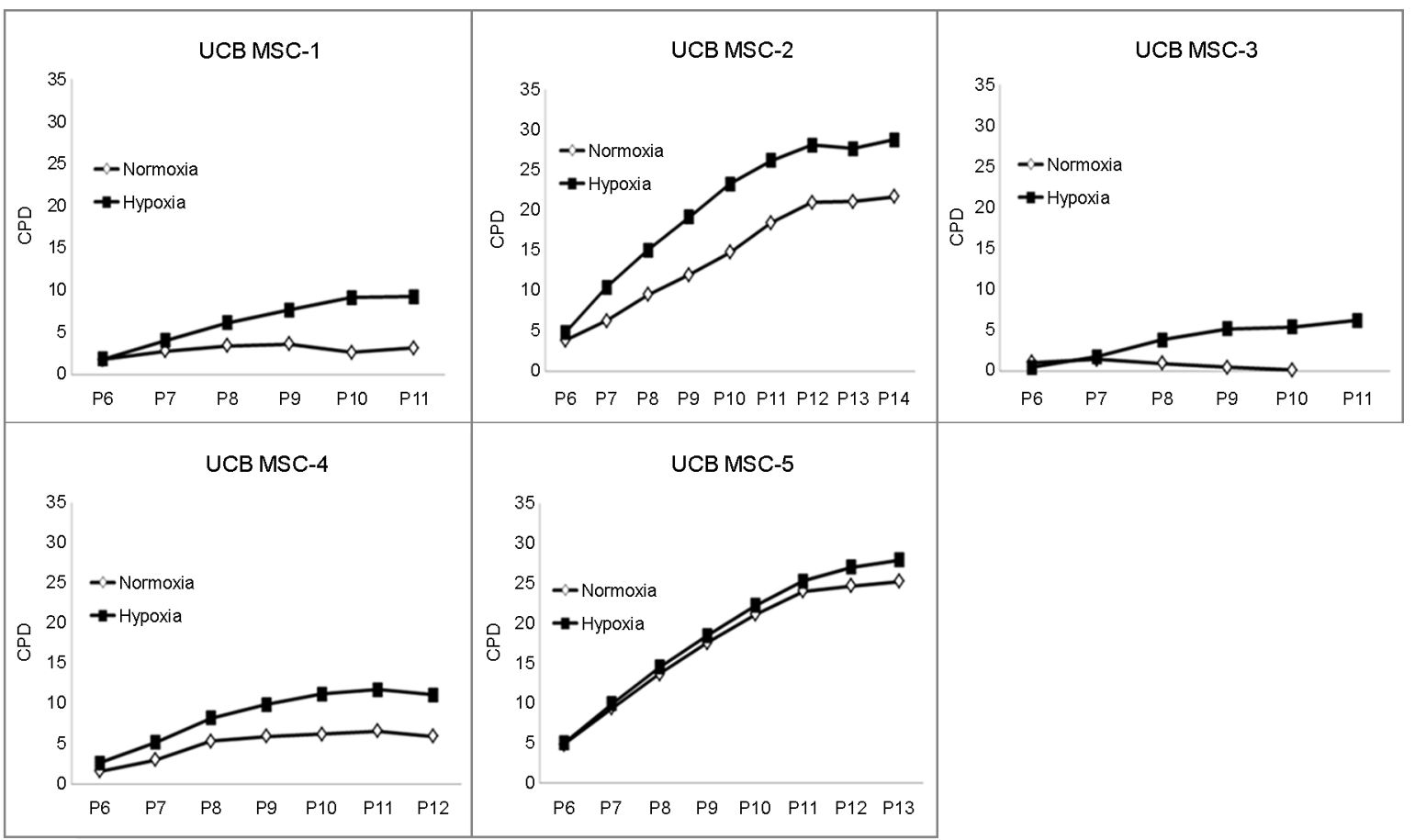

B

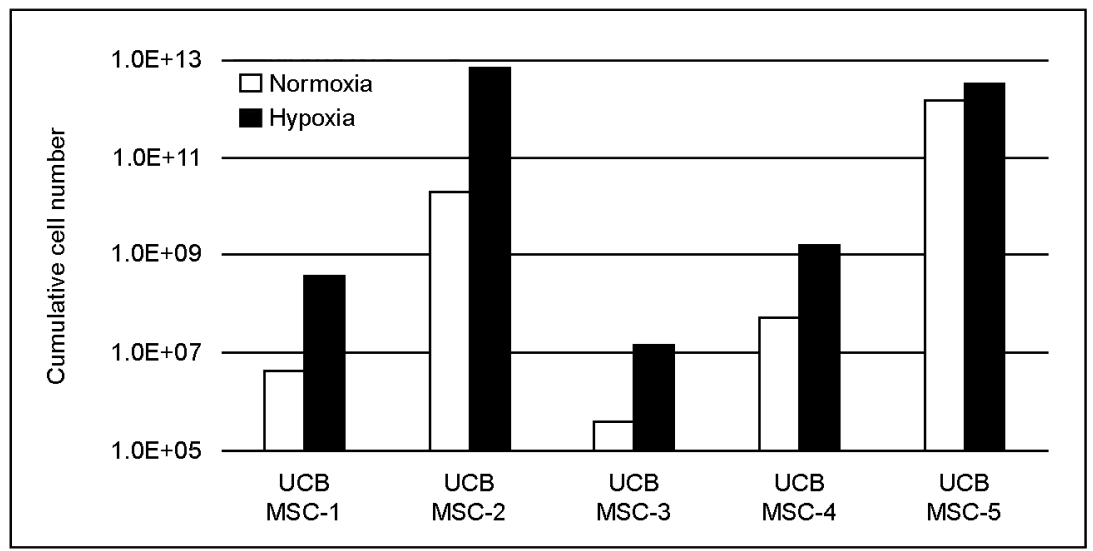

Fig. 1. Ex vivo expansion of UCBMSC cultured under hypoxia and normoxia. (A) Cumulative population doubling (CPD) of five UCBMSC samples cultured under hypoxic and normoxic conditions (P6-P14)

(B) Cumulative cell number at P10 of culture. $\mathrm{P}$ denotes passage number.

the five samples, our data suggest that hypoxia is more conducive to cell growth.

\section{Immunophenotypic characterization of UCB-MSC cultured under hypoxia and normoxia}

We assessed the isolated UCB-MSCs at P6 to ascertain the MSC phenotype. MSCs exhibit a specific immnunophenotype and are positive for specific surface markers and are negative for others. Using flow cytometry, we found that the UCB-MSCs at P6 exhibited the canonical immnunophenotype of a stem cell: positive expression of $\mathrm{CD}^{2} 9^{+}$, $\mathrm{CD}^{+} 3^{+}, \mathrm{CD} 0^{+}, \mathrm{CD} 105^{+}, \mathrm{CD} 166^{+}$and negative expression of $\mathrm{CD} 45^{+}, \mathrm{CD} 14^{+}, \mathrm{HLA}_{-\mathrm{DR}}^{+}$(Fig. 2). This result demonstrates that the isolated UCB-MSCs are of high purity. Next, we examined whether the UCB-MSCs cultured to the P9 stage still maintained their canonical MSC phenotype. We found that UCB-MSCs cultured under both hypoxia and normoxia maintained the MSC phenotype (Table 1). This 

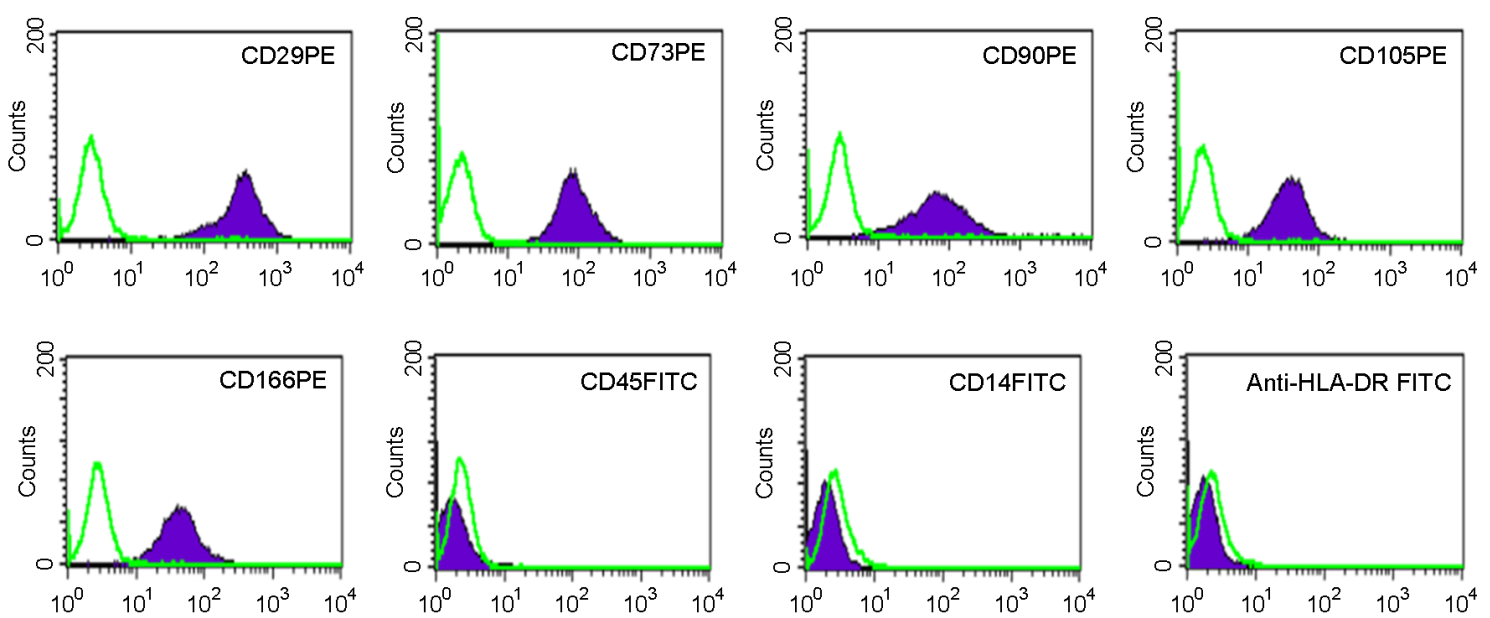

Fig. 2. Immunophenotypic analysis of UCB-MSCs. UCB-MSCs were cultured under hypoxic conditions and surface stained with specific antibodies. Staining intensity was examined by flow cytometry. Data are shown as histograms. Green, isotype matched control. Purple, specific antibodies. A representative histogram from one sample (UCB-MSC-3) at P6 is shown.

Table 1. Immunophenotypic characterization of the UCB-MSCs cultured under normoxia and hypoxia

\begin{tabular}{lcccccccc}
\hline \hline & CD29 & CD73 & CD90 & CD105 & CD166 & CD45 & CD14 & HLA-DR \\
\hline Hypoxia & $95.2 \pm 3.5$ & $96.8 \pm 1.9$ & $95.9 \pm 1.7$ & $95.7 \pm 5.4$ & $93.4 \pm 5.9$ & $0.3 \pm 0.2$ & $0.2 \pm 0.1$ & $0.3 \pm 0.3$ \\
Normoxia & $94.8 \pm 2.7$ & $97.3 \pm 2.5$ & $95.4 \pm 2.3$ & $94.9 \pm 6.1$ & $93.6 \pm 6.6$ & $0.2 \pm 0.1$ & $0.3 \pm 0.2$ & $0.2 \pm 0.1$ \\
\hline
\end{tabular}

Cells at P10 were examined by flow cytometry. Histograms from four samples were pooled for analysis. Percentages indicate positive staining when compared to isotype matched controls. Data are presented as the mean $\pm \operatorname{SD}(n=4)$.

result demonstrates that while the UCB-MSCs proliferate better in under hypoxia, the MSCs retain their phenotype.

\section{Osteogenic differentiation potential of UCB-MSC cultured under hypoxia and normoxia}

MSCs are known to progressively lose their ability to differentiate into other cell lineages during prolonged in vitro expansion. We examined whether UCB-MSCs retained their differentiation potential when expanded under hypoxia. UCB-MSCs at P10 were cultured in osteogenic differentiation media for two weeks and then stained with von Kossa or ALP. We found that cells expanded under hypoxic conditions differentiated similar to or better than cells expanded under normoxia (Fig. 3). These results suggest that the differentiation potential of hypoxic cells are more effective compared to normoxic cells.

\section{Cellular senescence of UCB-MSC cultured under hypoxia and normoxia}

One major concern of MSCs undergoing in vitro expansion is that prolonged expansion increases the frequency of cells undergoing cellular senescence. Since our data suggest that hypoxic cells expand faster compared to normoxic cells, we were concerned whether this increased expansion facilitated cellular senescence. Therefore, we compared cellular senescence in hypoxic cells compare to normoxic cells. UCB-MSCs which exhibited $<2$-fold expansion in between subcultures were examined for the presence of senescence associated $\beta$-galactosidase (SA $\beta$-gal) which is present in cells undergoing senescence (Fig. 4A) (Dimri et al., 1995). We found that in all five samples, cells expanded under hypoxia exhibited approximately $<50 \%$ senescent cells (Fig. 4B). These results suggest that hypoxic cells which expand 
A
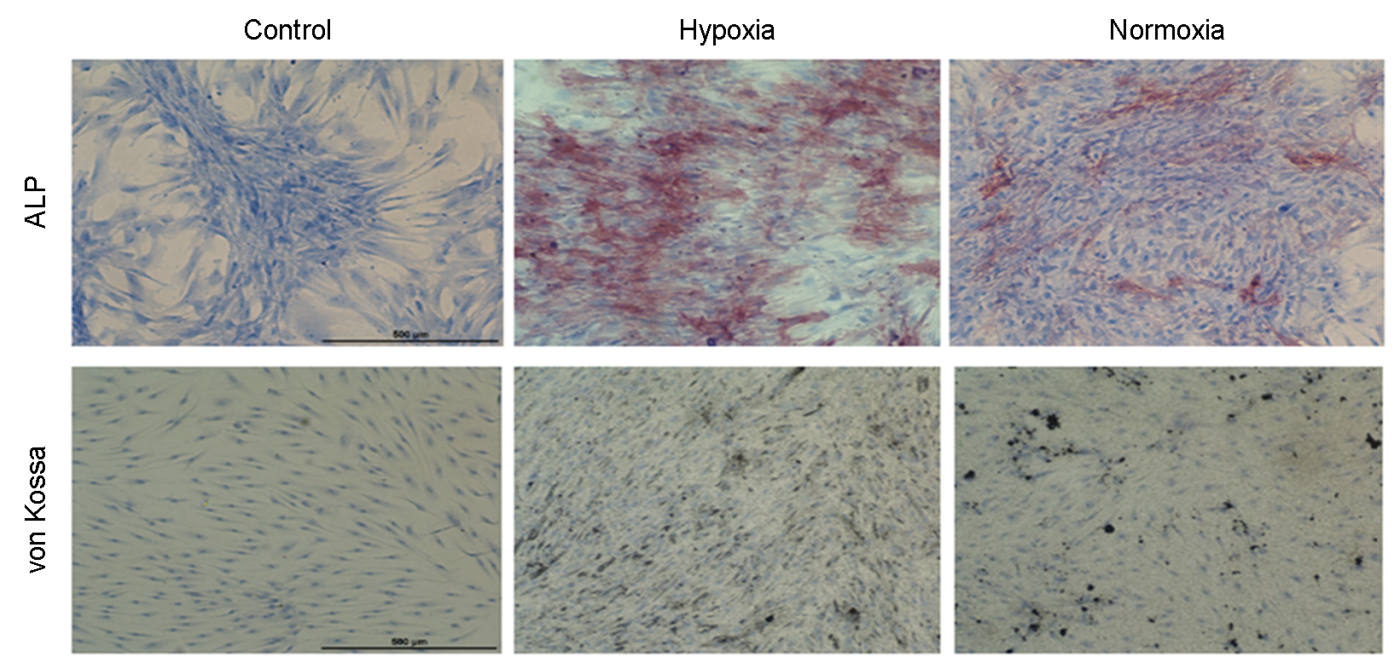

B

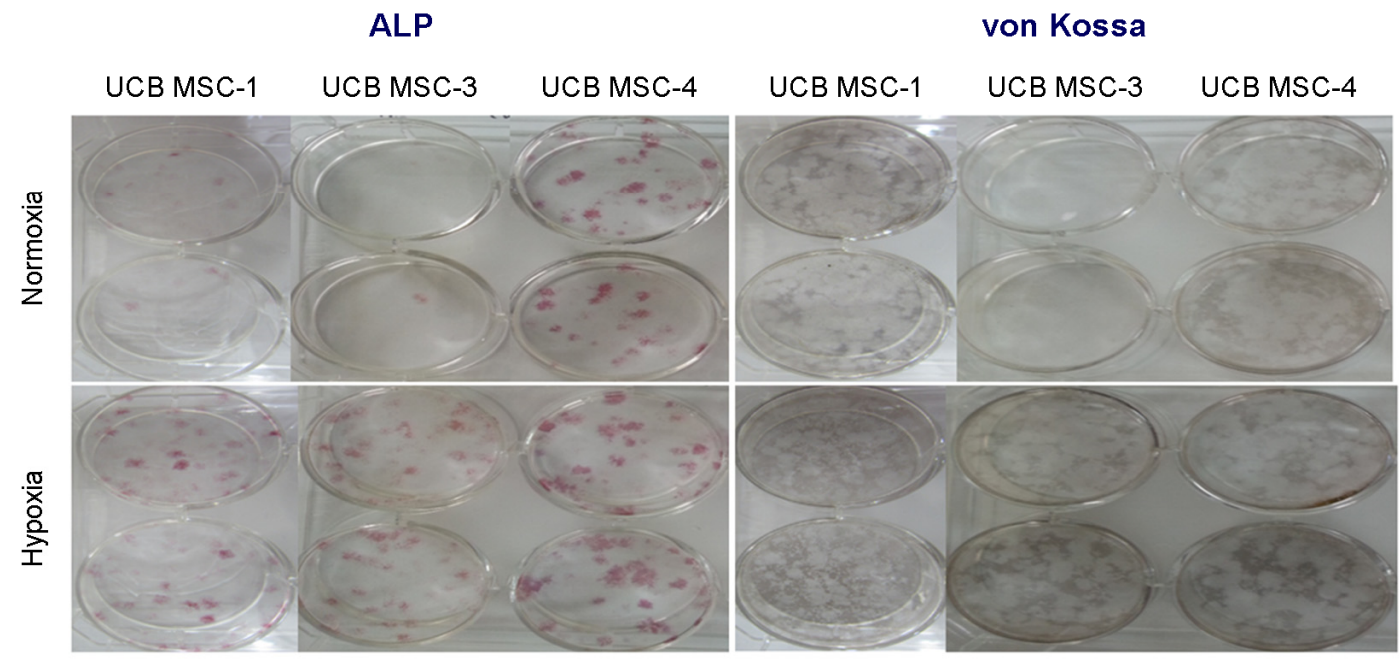

Fig. 3. Osteogenic differentiation of UCB-MSCs cultured under hypoxia and normoxia. UCB-MSCs (P10) were cultured under hypoxia or normoxia for two weeks in osteogenic induction media. Osteogenic cells were detected by alkaline phosphatase (red) or by von Kossa staining (black) and then counterstained with hematoxylin. (A) high magnification image of cells. (B) low magnification of cell culture wells. Scale bar $=500 \mu \mathrm{m}$. Control, hypoxic cells cultured in the absence of osteogenic induction media.

better than normoxic cells exhibit less cellular senescence compared to normoxic cells.

\section{Expression of stem cell specific genes in UCB-MSCs cultured under hypoxic and normoxic conditions}

Stem cells maintain their self-renewing capacity by continuous expression of select stemness-related transcriptional factors. Among these transcription factors, we examined the expression of OCT4, NANOG and SOX2 which are known to be expressed in MSCs having self-renewing capacity. We found increased mRNA levels of OCT4 (1.34 fold), NANOG (2.26 fold) and SOX2 (1.88 fold) in hypoxic cells compared to normoxic cells (Fig. 5). These results indicate that the stem cell regulatory genes are expressed at higher levels in UCB-MSCs expanded under hypoxic conditions suggesting that hypoxic expansion maintains stemness of cells. 
A
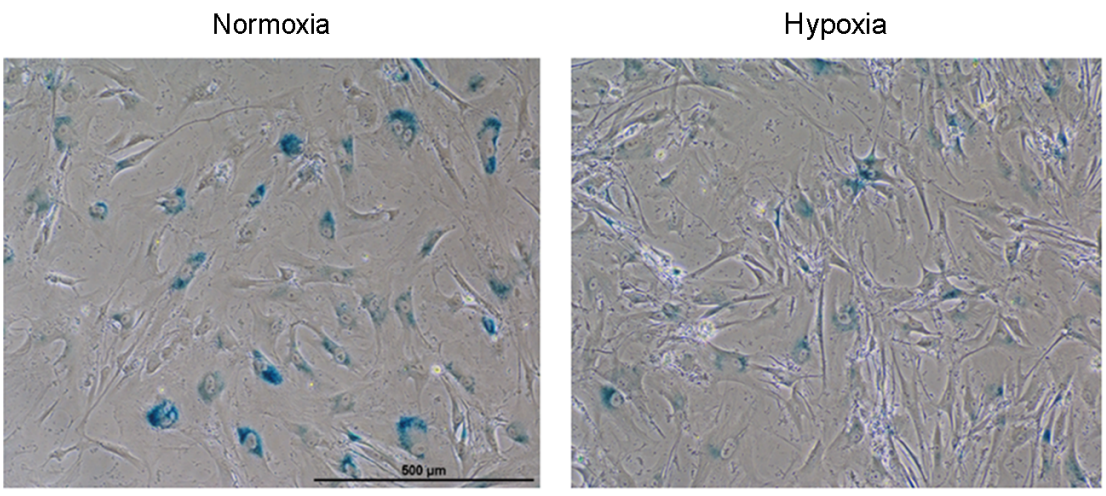

B

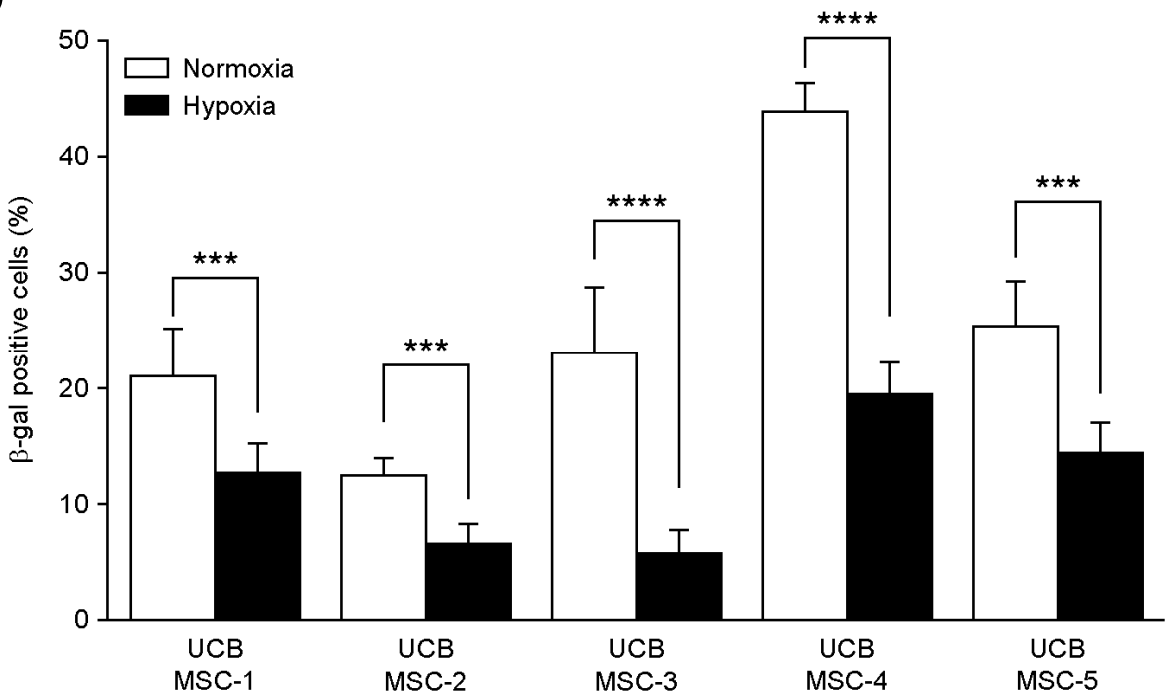

Fig. 4. Cellular senescence of UCB-MSCs cultured under hypoxia and normoxia. (A) $\beta$-galactosidase staining of UCB-MSC cultured under hypoxia and normoxia. Cells undergoing senescence are stained blue. Scale bar $=500 \mu \mathrm{m}$. (B) Quantification of $\beta$-gal positive cells. Cells were stained for $\beta$-gal, enumerated and data rendered by SIGMAPLOT. Cells were used as follows: UCB-MSC-1 (P11), UCB-MSC-2 (P13), UCB-MSC-3 (P8), UCB-MSC-4 (P11), UCB-MSC-5 (P11). Error bars represent the means \pm SD (***; $P<0.001, * * * ; P<0.0001)$.

\section{DISCUSSION}

MSCs, as well as hematopoietic stem cells and neural stem cells have been found to reside in a low oxygen environment or "niche". Culture of cells in this low oxygen environment called hypoxia ( $<5 \%$ oxygen tension) have been shown to enhance stem cell proliferation, decrease senescence and decrease cell death (Mohyeldin et al., 2010). Chow et al. the bone marrow niche is a low oxygen environment (Chow et al., 2001). Culture of bone marrow derived MSC at $\%$ oxygen tension exhibited increased proliferation and efficient energy metabolism whereas the differentiation potential was similar to normoxic cells (Dos Santos et al., 2010). In another study, hypoxia greatly influenced adipose tissue proliferation, metabolism and differentiation (Wang et al., 2005). Similar to these studies, we also found that UCB-MSCs cultured under hypoxic conditions maintained their self-renewal capacity and less senescence and improved cell proliferation similar to studies using MSCs isolated from other sources (Wang et al., 2005; Grayson et al., 2006; Grayson et al., 2007; Dos Santos et al., 2010). As for differentiation potential, UCB-MSC grown in hypoxia also 


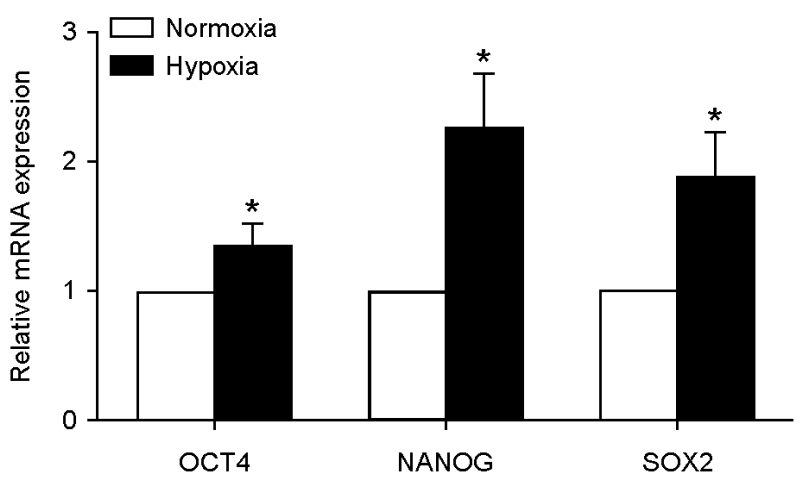

Fig. 5. Expression of OCT4, NANOG and SOX2 in UCB-MSCs cultured under normoxic and hypoxic conditions. UCB-MSCs (P8) were cultured under hypoxia or normoxia for 18 days and expression of OCT4, NANOG and SOX2 assessed by real-ime PCR. Data from UCB-MSCs under normoxic conditions were normalized to 1 . Error bars represent the means \pm SD. $n=3 . * P<0.05$.

had similar or greater differentiating ability compared to normoxic cells. This is in contrast to the results by Dos Santos et al. who showed that MSC isolated from the bone marrow did not show a difference in differentiation ability when cultured in hypoxic versus normoxic conditions (Dos Santos et al., 2010). This difference may reside in the source of the MSCs and other undefined culture parameters.

Stem cells require expression of several genes for maintenance of the stem cell phenotype such as OCT4, SOX2 and NANOG (Niwa et al., 2000; Silva et al., 2006; Babaie et al., 2007). When cells are subjected to hypoxic conditions, cells express HIF (hypoxia-inducible factor) dependent genes (Kaelin and Ratcliffe, 2008; Semenza, 2011). HIF is a ubiquitous found protein in all tissue types and during hypoxia, HIF regulates transcription of $>40$ genes all of which are involved in various aspects of oxygen transport, glucose energy metabolism, cell proliferation and survival (Wiener et al., 1996). It was determined that culturing bone marrow stem cells under hypoxic conditions resulted in induction of stem cell specific genes such as OCT4 and Rex-1 as well as extracellular matrix genes such as fibronectin. This observation was also found in stem cells isolated from the adipose tissues which exhibited an improvement in differentiation potential of adipose stem cells (Wang et al., 2005; Grayson et al., 2006; Grayson et al., 2007). Similar findings were found in human placenta derived MSC (Ni et al., 2014). During the preparation of this manuscript, Drela et al. found showed that low oxygen atmosphere facilitated proliferation and maintained undifferentiated state of UCB-MSCs (Drela et al., 2014). In this study, isolated UCB-MSCs were cultured in Wharton Jelly under hypoxic and normoxic conditions. They found that hypoxic cultures showed increased proliferation and induction of OCT4, SOX2, Rex-1 and NANOG. They also showed that spontaneous differentiation of UCB-MSCs into neural cells was inhibited by hypoxia. Although they did not show that the UCB-MSCs maintained their differentiation potential, these data are in agreement with our results.

To use MSCs for clinical therapy, a large number of cells are required and thus most stem cells are expanded in vitro for at least 3 weeks. However, for the cells to have therapeutic potential, the cells must remain undifferentiated and maintain self-renewal capacity and but able to differentiate into other cell lineage once placed into patients. We found that by culturing the UCB-MSCs under hypoxic conditions, the cells proliferation improved while maintaining differentiation potential. This procedure should provide a simple and enhanced method of quickly expanding UCBMSCs for therapy.

\section{REFERENCES}

Alves H, van Ginkel J, Groen N, Hulsman M, Mentink A, Reinders M, van Blitterswijk C, de Boer J. A mesenchymal stromal cell gene signature for donor age. PloS One. 2012. 7: e42908. Avanzini MA, Bernardo ME, Cometa AM, Perotti C, Zaffaroni N, Novara F, Visai L, Moretta A, Del Fante C, Villa R, Ball LM, Fibbe WE, Maccario R, Locatelli F. Generation of mesenchymal stromal cells in the presence of platelet lysate: a phenotypic and functional comparison of umbilical cord blood- and bone marrow-derived progenitors. Haematologica. 2009. 94: 1649-1660.

Babaie Y, Herwig R, Greber B, Brink TC, Wruck W, Groth D, Lehrach H, Burdon T, Adjaye J. Analysis of Oct4-dependent transcriptional networks regulating self-renewal and pluripotency in human embryonic stem cells. Stem Cells. 2007. 25: 500-510.

Braun RD, Lanzen JL, Snyder SA, Dewhirst MW. Comparison of tumor and normal tissue oxygen tension measurements using 
OxyLite or microelectrodes in rodents. Am J Physiol Heart Circ Physiol. 2001. 280: H2533-2544.

Caplan AI. Adult mesenchymal stem cells for tissue engineering versus regenerative medicine. J Cell Physiol. 2007. 213: 341 $-347$.

Chow DC, Wenning LA, Miller WM, Papoutsakis ET. Modeling $\mathrm{pO}_{2}$ distributions in the bone marrow hematopoietic compartment. II. Modified Kroghian models. Biophys J. 2001. 81: 685-696.

Cipolleschi MG, Dello Sbarba P, Olivotto M. The role of hypoxia in the maintenance of hematopoietic stem cells. Blood. 1993. 82: 2031-2037.

Dimri GP, Lee X, Basile G, Acosta M, Scott G, Roskelley C, Medrano EE, Linskens M, Rubelj I, Pereira-Smith O, Peacocke M, Campisi J. A biomarker that identifies senescent human cells in culture and in aging skin in vivo. Proc Natl Acad Sci USA. 1995. 92: 9363-9367.

Dos Santos F, Andrade PZ, Boura JS, Abecasis MM, da Silva CL, Cabral JM. Ex vivo expansion of human mesenchymal stem cells: a more effective cell proliferation kinetics and metabolism under hypoxia. J Cell Physiol. 2010. 223: 27-35.

Drela K, Sarnowska A, Siedlecka P, Szablowska-Gadomska I, Wielgos M, Jurga M, Lukomska B, Domanska-Janik K. Low oxygen atmosphere facilitates proliferation and maintains undifferentiated state of umbilical cord mesenchymal stem cells in an hypoxia inducible factor-dependent manner. Cytotherapy. 2014. 16: 881-892.

Erices A, Conget P, Minguell JJ. Mesenchymal progenitor cells in human umbilical cord blood. Br J Haematol. 2000. 109: 235 $-242$.

Fan X, Liu T, Liu Y, Ma X, Cui Z. Optimization of primary culture condition for mesenchymal stem cells derived from umbilical cord blood with factorial design. Biotechnol Prog. 2009. 25: 499-507.

Flynn A, Barry F, O'Brien T. UC blood-derived mesenchymal stromal cells: an overview. Cytotherapy. 2007. 9: 717-726.

Fukuchi Y, Nakajima H, Sugiyama D, Hirose I, Kitamura T, Tsuji K. Human placenta-derived cells have mesenchymal stem/ progenitor cell potential. Stem Cells. 2004. 22: 649-658.

Grayson WL, Zhao F, Bunnell B, Ma T. Hypoxia enhances proliferation and tissue formation of human mesenchymal stem cells. Biochem Biophys Res Commun. 2007. 358: 948-953.

Grayson WL, Zhao F, Izadpanah R, Bunnell B, Ma T. Effects of hypoxia on human mesenchymal stem cell expansion and plasticity in 3D constructs. J Cell Physiol. 2006. 207: 331-339.
Han K, Lee JE, Kwon SJ, Park SY, Shim SH, Kim H, Moon JH, Suh CS, Lim HJ. Human amnion-derived mesenchymal stem cells are a potential source for uterine stem cell therapy. Cell Prolif. 2008. 41: 709-725.

Jin HJ, Bae YK, Kim M, Kwon SJ, Jeon HB, Choi SJ, Kim SW, Yang YS, Oh W, Chang JW. Comparative analysis of human mesenchymal stem cells from bone marrow, adipose tissue, and umbilical cord blood as sources of cell therapy. Int $\mathrm{J}$ Med Sci. 2013. 14: 17986-18001.

Jo CH, Kim OS, Park EY, Kim BJ, Lee JH, Kang SB, Lee JH, Han HS, Rhee SH, Yoon KS. Fetal mesenchymal stem cells derived from human umbilical cord sustain primitive characteristics during extensive expansion. Cell Tissue Res. 2008. 334: 423-433.

Kaelin WG, Jr., Ratcliffe PJ. Oxygen sensing by metazoans: the central role of the HIF hydroxylase pathway. Mol Cell. 2008. 30: $393-402$

Kern S, Eichler H, Stoeve J, Kluter H, Bieback K. Comparative analysis of mesenchymal stem cells from bone marrow, umbilical cord blood, or adipose tissue. Stem Cells. 2006. 24: 1294-1301.

Kretlow JD, Jin YQ, Liu W, Zhang WJ, Hong TH, Zhou G, Baggett LS, Mikos AG, Cao Y. Donor age and cell passage affects differentiation potential of murine bone marrowderived stem cells. BMC Cell Biol. 2008. 9: 60.

Lee OK, Kuo TK, Chen WM, Lee KD, Hsieh SL, Chen TH. Isolation of multipotent mesenchymal stem cells from umbilical cord blood. Blood. 2004. 103: 1669-1675.

Lu LL, Liu YJ, Yang SG, Zhao QJ, Wang X, Gong W, Han ZB, $\mathrm{Xu}$ ZS, Lu YX, Liu D, Chen ZZ, Han ZC. Isolation and characterization of human umbilical cord mesenchymal stem cells with hematopoiesis-supportive function and other potentials. Haematologica. 2006. 91: 1017-1026.

Mohyeldin A, Garzon-Muvdi T, Quinones-Hinojosa A. Oxygen in stem cell biology: a critical component of the stem cell niche. Cell Stem Cell. 2010. 7: 150-161.

Mosna F, Sensebe L, Krampera M. Human bone marrow and adipose tissue mesenchymal stem cells: a user's guide. Stem Cells Dev. 2010. 19: 1449-1470.

Ni L, Liu X, Sochacki KR, Ebraheim M, Fahrenkopf M, Shi Q, Liu J, Yang H. Effects of hypoxia on differentiation from human placenta-derived mesenchymal stem cells to nucleus pulposus-like cells. Spine J. 2014. 14: 2451-2458.

Niwa H, Miyazaki J, Smith AG. Quantitative expression of Oct-3/4 defines differentiation, dedifferentiation or self-renewal of 
ES cells. Nature Genet. 2000. 24: 372-376.

Oh W, Kim DS, Yang YS, Lee JK. Immunological properties of umbilical cord blood-derived mesenchymal stromal cells. Cell Immunol. 2008. 251: 116-123.

Reinisch A, Bartmann C, Rohde E, Schallmoser K, Bjelic-Radisic V, Lanzer G, Linkesch W, Strunk D. Humanized system to propagate cord blood-derived multipotent mesenchymal stromal cells for clinical application. Regen Med. 2007. 2: 371-382.

Semenza GL. Oxygen sensing, homeostasis, and disease. New
Engl J Med. 2011. 365: 537-547.

Silva J, Chambers I, Pollard S, Smith A. Nanog promotes transfer of pluripotency after cell fusion. Nature. 2006. 441: 997-1001.

Wang DW, Fermor B, Gimble JM, Awad HA, Guilak F. Influence of oxygen on the proliferation and metabolism of adipose derived adult stem cells. J Cell Physiol. 2005. 204: 184-191.

Wiener CM, Booth G, Semenza GL. In vivo expression of mRNAs encoding hypoxia-inducible factor 1. Biochem Biophys Res Commun. 1996. 225: 485-488. 